\title{
Upregulation of the eIF4E signaling pathway contributes to the progression of gastric cancer, and targeting eIF4E by perifosine inhibits cell growth
}

\author{
SONG LIANG $^{1 *}$, RENHUA GUO $^{2 *}$, ZHIHONG ZHANG ${ }^{3 *}$, DONGXIAO LIU ${ }^{4}$, \\ HAO XU ${ }^{1}$, ZEKUAN XU ${ }^{1}$, XUERONG WANG ${ }^{5}$ and LI YANG $^{1}$
}

Departments of ${ }^{1}$ General Surgery, ${ }^{2}$ Medical Oncology and ${ }^{3}$ Pathology, The First Affiliated Hospital of Nanjing Medical University, Nanjing; ${ }^{4}$ Department of General Surgery, The Second Affiliated Hospital of South-east University, The Second Hospital of Nanjing, Nanjing; ${ }^{5}$ Department of Pharmacology, Nanjing Medical University, Nanjing, Jiangsu 210029, P.R. China

Received January 21, 2013; Accepted March 15, 2013

DOI: 10.3892/or.2013.2397

\begin{abstract}
The increase of eukaryotic translation initiation factor 4E (eIF4E) expression is frequently observed in several types of cancer, making eIF4E an attractive anticancer drug target. However, the role of eIF4E in gastric cancer pathogenesis remains unclear. Perifosine is a bioavailable alkylphospholipid exhibiting antitumor activity in a series of cancer types. In this study, gastric cancer cell lines were selected to explore the role of eIF4E as a potential target for treating human gastric cancer. The expression of total eIF4E (T-eIF4E)and phosphorylated eIF4E (p-eIF4E) in gastric cancer samples was detected by immunohistochemical assay. RNA interference was used to silence eIF4E expression. Sulforhodamine B assay was performed to evaluate tumor cell viability. Colony formation assay was used to examine the effects of eIF4E small interfering RNA (siRNA) or perifosine on colony formation. The mRNA levels of eIF4E were analyzed by qRT-PCR and western blot analysis was carried out to evaluate the expression of Akt and eIF4E. The results showed that increased expression levels of T-eIF4E and p-eIF4E were found in
\end{abstract}

Correspondence to: Dr Li Yang, Department of General Surgery, The First Affiliated Hospital of Nanjing Medical University, 300 Guangzhou Road, Nanjing, Jiangsu 210029, P.R. China E-mail: pwkyangli@yahoo.com.cn

Professor Xuerong Wang, Department of Pharmacology, Nanjing Medical University, Nanjing, Jiangsu 210029, P.R. China

E-mail: wangxrnj@gmail.com

*Contributed equally

Abbreviations: eIF4E, eukaryotic translation initiation factor 4E; p-eIF4E, phosphorylated eIF4E; siRNA, small interfering RNA; SRB, sulforhodamine B

Key words: eukaryotic translation initiation factor 4E, gastric cancer, perifosine, Akt gastric cancer tissues and cells. Reduced eIF4E expression blocked the proliferation of gastric cancer cells. Perifosine downregulated the T-eIF4E and p-eIF4E levels in a dose- and time-dependent manner; it also inhibited the growth of gastric cancer cells. Moreover, this inhibitory effect was significantly enhanced by the combination of eIF4E siRNA and perifosine treatments. Our results indicate that eIF4E gene silencing can inhibit tumor cell growth, and eIF4E can be developed as a promising therapeutic target for gastric cancer.

\section{Introduction}

Gastric cancer, a leading cause of cancer-related mortality worldwide, is the fourth most frequent malignancy. Despite marked improvements in surgical, chemo-, radio- and other adjuvant therapies, the 5-year survival rate of patients at the advanced stage remains $<20-25 \%(1,2)$. In recent years, emerging evidence has revealed various genetic changes involved in the progression of gastric cancer. It is critical to investigate the precise molecular mechanism of gastric cancer development for improved anticancer therapeutics.

Eukaryotic translation initiation factor 4E (eIF4E) plays a crucial role in several human tumors, including breast, head and neck, bladder, cervical, lung and prostate cancer (2). Enhanced eIF4E function resulting from eIF4E overexpression or activation of the Ras and phosphatidylinositol 3-kinase/ Akt pathways can selectively upregulate the translation initiation of cancer-related mRNA, such as c-Myc, cyclin D1 and Bcl-2 for tumor growth, angiogenesis and cell survival (3-5). The overexpression or knockdown of eIF4E provides insights into its functional significance in tumorigenesis. Knockdown of eIF4E can suppress proliferation and angiogenesis $(1,6)$. Additionally, elevated eIF4E expression confers resistance to multiple chemotherapy agents. A combination of chemotherapy with eIF4E silencing was reported to increase sensitivity to chemotherapeutic drugs such as cryptotanshinone, cisplatin, adriamycin, paclitaxel and docetaxel $(4,7,8)$.

Perifosine is a synthetic alkylphosphocholine (9), exhibiting antitumor activity through blocking cell membrane recruitment of the N-terminal Akt pleckstrin homology $(\mathrm{PH})$ 
domain. It also exerts an antitumor effect through MAPK pathway inhibition while inducing c-Jun NH2-terminal kinase (JNK) and upregulating death receptor 5 (DR5) (10-12). The clinical efficacy of perifosine was evaluated in a phase I clinical trial in patients with advanced tumor. Perifosine showed potent anticancer efficacy in a various types of cancer, including breast, prostate and renal cell cancer (13-15). However, there has been no report on the effect of perifosine against human gastric cancer cells, and its molecular mechanism has yet to be fully elucidated.

In the present study, we first examined the expression of total eIF4E (T-eIF4E) and phosphorylated eIF4E (p-eIF4E) in human tissues and gastric cancer cell lines, and analyzed the correlation between T-eIF4E and p-eIF4E levels and the clinicopathological characteristics of gastric cancer. We then discussed the proliferation of human gastric cancer SGC7901 and MGC803 cells following eIF4E gene silencing. Furthermore, we investigated the eIF4E signaling pathway regulated by perifosine and the growth inhibitory effect of perifosine on gastric cancer cells. We further identified a combined effect of eIF4E small interfering RNA (siRNA) and perifosine on the growth of gastric cancer cells. These findings identified the oncogenic function of eIF4E and presented the possibility of eIF4E as an effective antitumor target in gastric cancer.

\section{Materials and methods}

Reagents. Perifosine was purchased from Selleck Chemicals LLC (Houston, TX, USA) and was dissolved in PBS to make a $50 \mathrm{mmol} / 1$ stock solution and stored at $-20^{\circ} \mathrm{C}$. RPMI-1640 culture medium was purchased from Gibco-BRL (Grand Island, NY, USA). Sulforhodamine B (SRB) was purchased from Sigma Chemical Co. The Lipofectamine 2000 transfection reagent was purchased from Invitrogen Life Technologies (Carlsbad, CA, USA). Rabbit polyclonal antibodies against p-Akt (s473) (9271) and eIF4E (9742), and rabbit monoclonal antibody against Akt (9272) were purchased from Cell Signaling Technology, Inc. (Beverly, MA, USA). Rabbit monoclonal antibody against actin (sc-130300) was purchased from Santa Cruz Biotechnology, Inc. (Santa Cruz, CA, USA). Rabbit polyclonal anti-GAPDH (AP0063) was purchased from Bioworld Technology Inc. (Louis Park, MN, USA). Rabbit monoclonal antibody against p-eIF4E (s209) (2227-1) was purchased from Epitomics Inc. (Burlingame, CA, USA).

Human tissue samples and immunohistochemical assay. The tissues used in this study were obtained from 42 gastric cancer samples and 42 adjacent normal mucosal tissues from patients (32 men and 10 women; age range, 31-77 years) who underwent curative gastrectomy at the First Affiliated Hospital of Nanjing Medical University since 2012. The histologic types and staging of the gastric cancer samples were recorded in accordance with the Lauren and TNM classifications (proposed by the American Joint Committee, 2010 version) respectively. None of the patients had conducted routine treatment including chemotherapy and radiotherapy. Immunohistochemical staining was conducted on serial sections for formalin-fixed and paraffin-embedded tissues. The T-eIF4E and p-eIF4E were detected using a labelled streptavidin biotin (LSAB) method following autoclave antigen retrieval. The slides were then incubated with $3 \%$ hydrogen peroxide for $15 \mathrm{~min}$ to block endogenous peroxidase activity. Immunohistochemical staining was performed on a BenchMark XT (Ventana Medical Systems, Inc., Tucson, AZ, USA) according to the manufacturer's instructions. Finally the slides were lightly counterstained with hematoxylin. Immunohistochemical staining was assessed according to the immunoreactive score (IRS) that evaluated the staining intensity and the proportion of positive tumor cells. The staining intensity was graded as 0 (no staining), 1 (light yellow), 2 (yellow) and 3 (dark yellow). The proportion of positive tumor cells was scored as 0 (negative), $1(<10 \%), 2(10-50 \%)$, and $3(>50 \%)$. The two scores were multiplied and the IRS was determined: values $\geq 3$ were defined as cytoplasmic expression positive, and values $<3$ were regarded as negative.

Cells and cell culture. The gastric cancer cell lines SGC7901, MGC803, AGS and MKN45 were obtained from the Shanghai Institutes for Biological Sciences, Chinese Academy of Sciences, China. The cells were cultured in RPMI-1640 medium containing 10\% fetal bovine serum (Gibco-BRL; FBS) at $37^{\circ} \mathrm{C}$ in an incubator containing $95 \%$ air and $5 \% \mathrm{CO}_{2}$.

RNA interference. The oligonucleotides of siRNA targeting eIF4e (5'-AAGGACGATGGCTAATTACAT-3') and a nontargeting control were chemically designed and synthesized by Invitrogen Life Technologies. MGC803 and SGC7901 cells were seeded in 6-well plates at a density of $3.6 \times 10^{5}$ cells/well and transfected with $100 \mathrm{nmol} / \mathrm{l}$ eIF4e or control siRNAs for 24 h using Lipofectamine 2000 (Invitrogen Life Technologies) according to the manufacturer's instructions. The cells were reseeded to 96-well plates and then treated with perifosine for a further 3 days for SRB assay. The cells transfected with the above siRNAs for $48 \mathrm{~h}$ were harvested for total RNA, the purification of whole protein lysates, quantitative real-time PCR (qRT-PCR) assay and western blot analysis.

$S R B$ assay. Gastric cancer cells $\left(3 \times 10^{3}\right)$ were plated in 96-well plates for $24 \mathrm{~h}$ and then treated with perifosine (0.125-15 $\mu \mathrm{mol} / \mathrm{l})$. The cells following RNA interference were seeded in 96 -well plates $\left(1.5 \times 10^{3}\right.$ cells/well $)$ and treated with different concentrations of perifosine for $72 \mathrm{~h}$. Monolayer cells were fixed and stained with $0.4 \%$ (w/v) SRB. Finally, the dye was dissolved in $10 \mathrm{mM}$ Tris base solution for $5 \mathrm{~min}$ at room temperature with agitation. Absorbance was measured at $500 \mathrm{~nm}$ using a $\mu$ Quant Universal Microplate Spectrophotometer (BioTek Instruments, Inc.).

Colony formation assay. MGC803 and SGC7901 cells were seeded in 24-well plates for $24 \mathrm{~h}$ and then exposed to different concentrations of perifosine $(0.25-15 \mu \mathrm{mol} / \mathrm{l})$. After cultivating in RPMI-1640 medium containing $10 \%$ FBS for 12 days, the resulting cell colonies were fixed and stained with $0.5 \%$ crystal violet for $10 \mathrm{~min}$. All colonies visible with the naked eye (>50 cells) were counted individually and their colony formation rates were evaluated (clone formation rate $=$ the number of clones/well/100). Each clone was repeated in triplicate.

Two days after siRNA transfection, 100 cells from each of the siRNA, negative control and blank groups were seeded in 
24-well plates and cultured in $3 \mathrm{ml}$ RPMI-1640 medium with $10 \%$ FBS for 12 days. All colonies visible with the naked eye were counted individually and the clone formation rates were evaluated.

$q R T-P C R$. Total RNA was extracted using TRIzol reagent (Invitrogen Life Technologies) according to the manufacturer's instructions and treated with RNase-free DNase. Then, cDNA was prepared using M-MLV reverse transcriptase (Promega, Madison, WI, USA). qRT-PCR analysis was performed using a Power SYBR-Green PCR Master Mix from Applied Biosystems, Inc. (Foster City, CA, USA) under the following conditions: $10 \mathrm{~min}$ at $95^{\circ} \mathrm{C}$ followed by 40 cycles at $95^{\circ} \mathrm{C}$ for $15 \mathrm{sec}, 60^{\circ} \mathrm{C}$ for $1 \mathrm{~min}$ using the ABI PRISM 7300 sequence detection system of Applied Biosystems Inc. The relative expression levels of target genes were calculated through normalizing to the expression of the control gene GAPDH in each experiment.

The sequences of specific primers were: eIF4E, 5'-CCT ACAGAACAGATGGGCACTC-3' (forward) and 5'-GCC CAAAAGTCTTCAACAGTATCA-3' (reverse); GAPDH, 5'-ATGGGGAAGGTGAAGGTCG-3' (forward) and 5'-GGG GTCATTGATGGCAACAATA-3' (reverse) and were designed and synthesized by Invitrogen Life Technologies, Inc.

Western blot analysis. RIPA lysis buffer was purchased from Cell Signaling Technology, Inc. and was stored at $4{ }^{\circ} \mathrm{C}$. Protease inhibitors were added prior to use. Cells were harvested after treatment and subjected to western blot analysis as previously described (16).

Statistical analysis. All data from three independent experiments are expressed as the means \pm SD. Differences between the groups were assessed by two-sided unpaired Student's t-test. The Fisher's exact test or the $\chi^{2}$ test was used to test the correlation between eIF4E expression and clinicopathological parameters. $\mathrm{P}<0.05$ was considered to indicate a statistically significant difference. The $\mathrm{IC}_{50}$ value of perifosine was calculated by Bliss software.

\section{Results}

Expression of T-eIF4E and p-eIF4E correlates with the clinicopathological parameters of gastric cancer. Due to the frequently elevated level of eIF4E in the progression of human cancer (17), we examined the expression of T-eIF4E and p-eIF4E in gastric cancer samples (Fig. 1). T-eIF4E and p-eIF4E were overexpressed in gastric tumor tissues compared with adjacent non-cancerous tissues. The positive rate of T-eIF4E and p-eIF4E was 78.57\% (33/42) and 76.19\% (32/42), respectively. T-eIF4E and p-eIF4E were mainly seen in the cytoplasm of primary cancer cells. Table I shows the correlation between T-eIF4E and p-eIF4E expression and clinicopathological parameters in gastric cancer. A significant correlation was found between T-eIF4E overexpression and distance metastasis in patients $(\mathrm{P}=0.026)$ (Table I), which was consistent with previous studies (2). The results indicated eIF4E gene expression may be involved in the development and could be used as an independent prognosis marker in patients with gastric cancer.
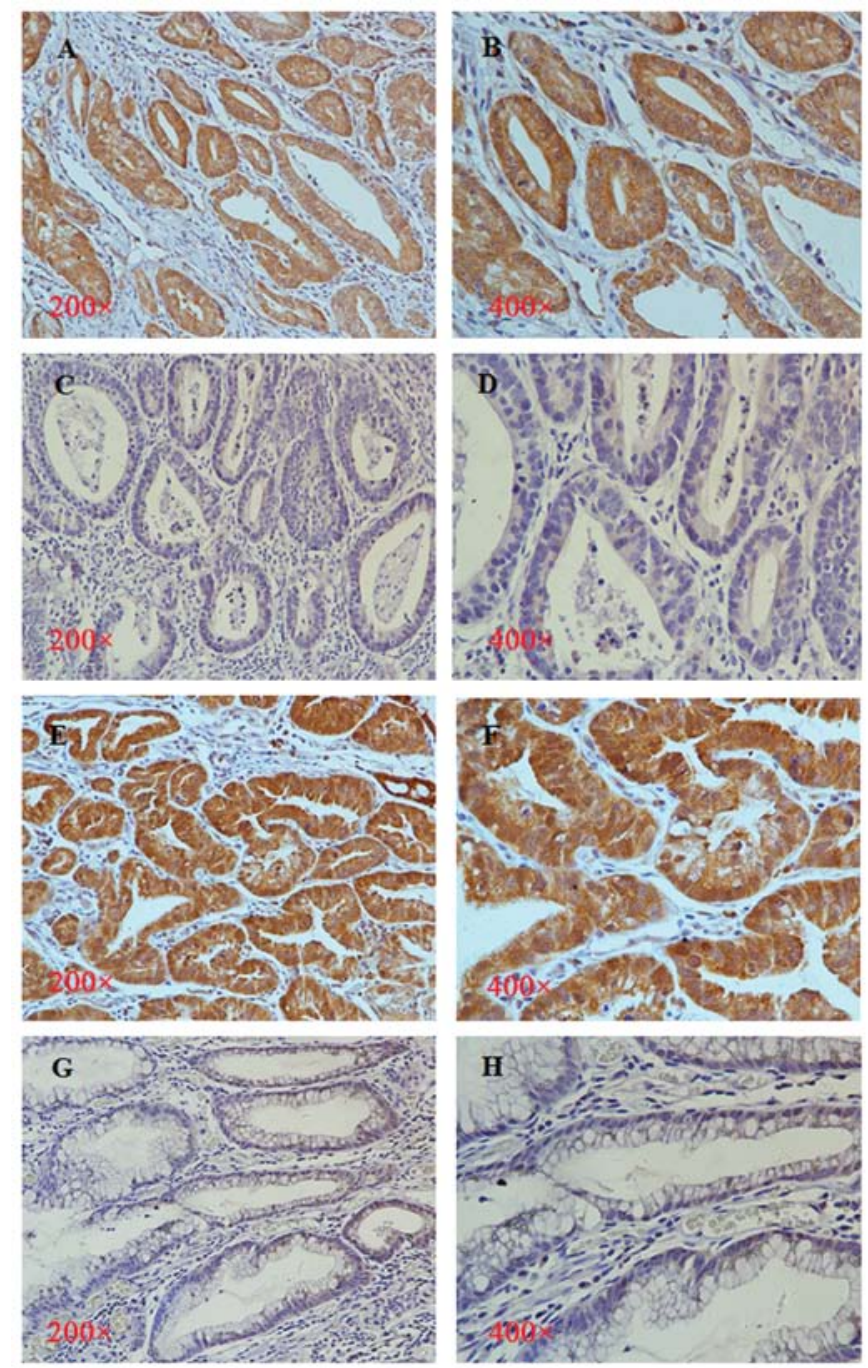

Figure 1. Immunohistochemical staining of T-eIF4E and p-eIF4E in the nontumorous mucosal and tumor tissues of gastric cancer patients. T-eIF4E was overexpressed in gastric cancer samples, original magnification, (A) x200, (B) $x 400$. The staining of T-eIF4E was weak in normal gastric mucosa, original magnification, (C) x200, (D) x400. The overexpression of p-eIF4E was found in gastric cancer tissues, original magnification, (E) x200, (F) x400. The staining of $\mathrm{p}$-eIF4E was weak in normal gastric mucosa, original magnification, (G) x200, (H) x400.

Expression of eIF4E is higher in gastric cancer cells than in human gastric epithelial cells. To further investigate the relationship between elevated eIF4E levels and gastric cancer, we examined the mRNA and protein levels of eIF4E in gastric cancer SGC7901 and MGC803 cells, as well as human gastric epithelial cells (GES-1) using qRT-PCR assay and western blot analysis. The expression of eIF4E mRNA was higher in gastric cancer cells than in GES-1 cells (Fig. 2A). Western blot analysis showed that T-eIF4E and p-eIF4E expression levels were considerably higher in gastric cancer cells than in GES-1 (Fig. 2B). These results indicated that eIF4E functions as an oncogene in gastric cancer.

Downregulation of eIF4E expression reduces the growth of gastric cancer cells. To investigate the potential functions of eIF4E gene in gastric cancer, we first explored the effect of eIF4E silencing on the growth of MGC803 and SGC7901 cells. 
Table I. The correlation between T-eIF4E and p-eIF4E expression and the clinicopathological characteristics in gastric cancer patients.

\begin{tabular}{|c|c|c|c|c|c|c|c|}
\hline \multirow[b]{2}{*}{ Characteristics } & \multirow[b]{2}{*}{ Total } & \multicolumn{2}{|c|}{ T-eIF4E } & \multirow[b]{2}{*}{ P-value } & \multicolumn{2}{|c|}{ p-eIF4E } & \multirow[b]{2}{*}{ P-value } \\
\hline & & - & + & & - & + & \\
\hline \multicolumn{8}{|l|}{ Age } \\
\hline$<60$ & 20 & 4 & 16 & 1.000 & 4 & 16 & 0.849 \\
\hline$\geq 60$ & 22 & 5 & 17 & & 6 & 16 & \\
\hline \multicolumn{8}{|l|}{ Gender } \\
\hline Male & 32 & 6 & 26 & 0.753 & 9 & 23 & 0.454 \\
\hline Female & 10 & 3 & 7 & & 1 & 9 & \\
\hline \multicolumn{8}{|l|}{ Tumor size } \\
\hline$\leq 5 \mathrm{~cm}$ & 27 & 6 & 21 & 1.000 & 8 & 19 & 0.418 \\
\hline$>5 \mathrm{~cm}$ & 15 & 3 & 12 & & 2 & 13 & \\
\hline \multicolumn{8}{|l|}{ TNM stage ${ }^{a}$} \\
\hline I, II & 15 & 4 & 11 & 0.823 & 6 & 9 & 0.145 \\
\hline III, IV & 27 & 5 & 22 & & 4 & 23 & \\
\hline \multicolumn{8}{|c|}{ Depth of invasion (T) } \\
\hline $\mathrm{T} 1, \mathrm{~T} 2$ & 15 & 4 & 11 & 0.823 & 6 & 9 & 0.145 \\
\hline $\mathrm{T} 3, \mathrm{~T} 4$ & 27 & 5 & 22 & & 4 & 23 & \\
\hline \multicolumn{8}{|c|}{ Lymph node metastasis (N) } \\
\hline N0 & 15 & 4 & 11 & 0.823 & 5 & 10 & 0.483 \\
\hline $\mathrm{N} 1-3$ & 27 & 5 & 22 & & 5 & 22 & \\
\hline \multicolumn{8}{|c|}{ Distant metastasis (M) } \\
\hline Yes & 4 & 3 & 1 & 0.026 & 1 & 3 & 1.000 \\
\hline No & 38 & 6 & 32 & & 9 & 29 & \\
\hline
\end{tabular}

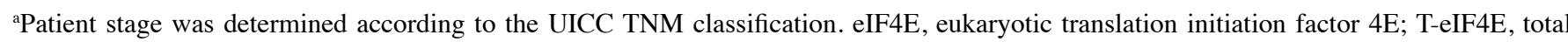
eIF4E; p-eIF4E, phosphorylated eIF4E.

A

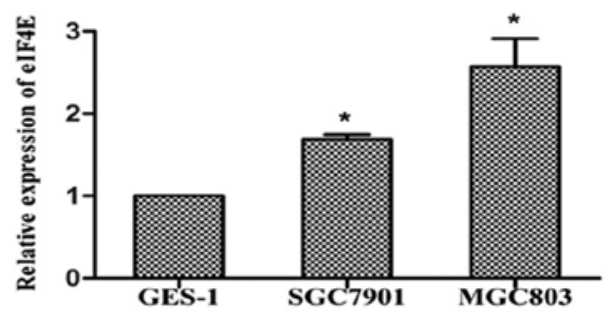

B

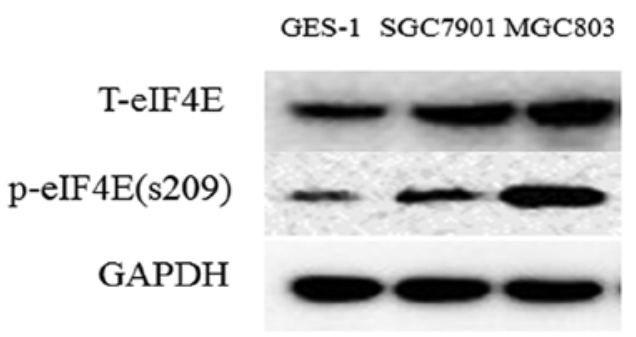

Figure 2. The expression of eIF4E before and after gene-silencing in gastric cancer cell lines. (A) Total RNA was extracted and the level of eIF4E mRNA in gastric epithelial cell line (GES-1) and gastric cancer cell lines (SGC7901 and MGC803) was analyzed by qRT-PCR. (B) Total protein was extracted and the expression levels of T-eIF4E and p-eIF4E were detected by western blot analysis using indicated antibodies in gastric cancer cells and GES-1 cells. All data in the different experimental groups are expressed as the means $\pm \mathrm{SD},{ }^{*} \mathrm{P}<0.05$.
In the qRT-PCR assay, a significantly decreased level of eIF4E mRNA was observed after $48 \mathrm{~h}$ transfection of $100 \mathrm{nmol} / \mathrm{l}$ eIF4E siRNA in comparison with a control siRNA in MGC803 and SGC7901 cells (Fig. 3A). Western blot analysis confirmed a significantly decreased level of T-eIF4E and p-eIF4E (s209), indicating a successful eIF4E knockdown (Fig. 3B). Our results showed that the gastric cancer cells with eIF4E siRNA exhibited markedly higher growth inhibition rates compared with the cells with a control siRNA $(\mathrm{P}<0.01)$ in a 5-day SRB assay (Fig. 3C and D), which was consistent with previous reports on breast cancer, primary central nervous system lymphoma cells $(18,19)$. Moreover, such inhibitory effects were also seen when the cells were transfected with eIF4E siRNA in a 10-day colony formation assay (Fig. 3E and F) and its colony formation rate was significantly lower in comparison with the control group. The result indicated that downregulation of eIF4E expression reduced the possibility of colony formation.

Perifosine decreases eIF4E mRNA level and inhibits the Akt eIF4E signaling pathway while downregulating T-eIF4E and p-eIF4E protein expression. Due to the crucial role of eIF4E in the growth of gastric cancer, we further examined the levels of eIF4E following perifosine (an Akt inhibitor) treatment. We explored whether perifosine reduced the proliferation 
A

MGC803

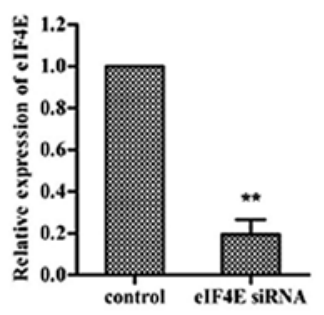

B

SGC7901

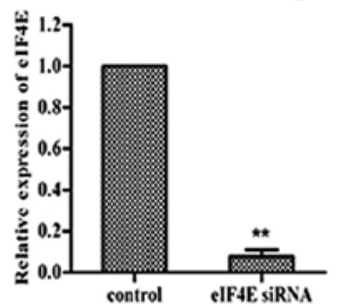

p-eIF4E(s209)

$\beta$-actin

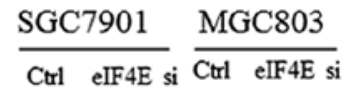

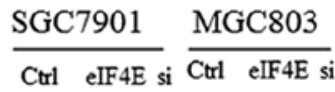

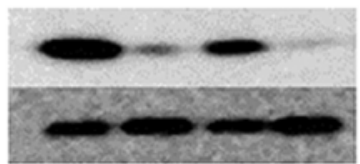

SGC7901 MGC803

\section{T-eIF4E} $\beta$-actin

C

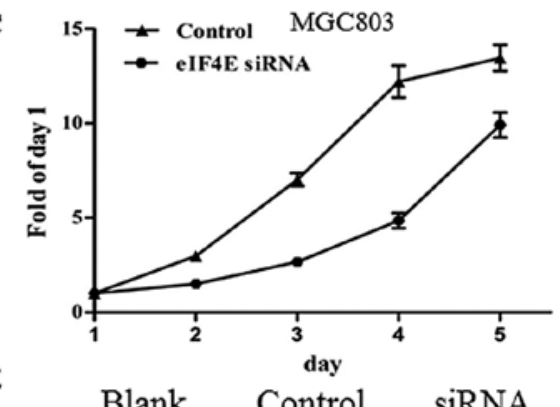

E
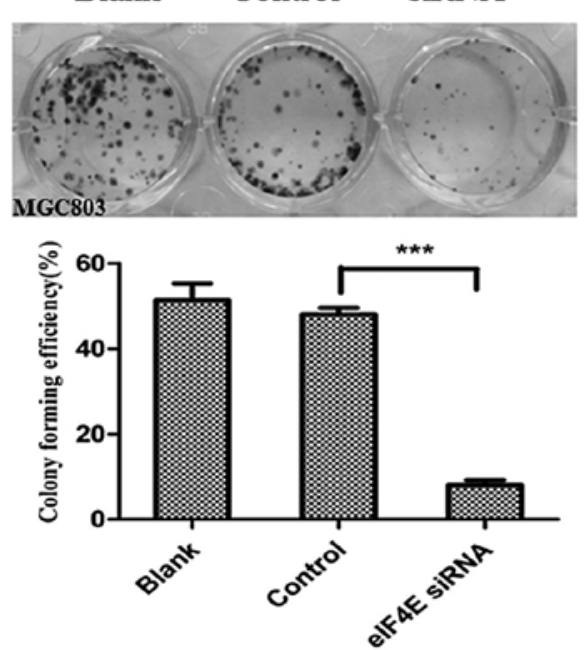

D

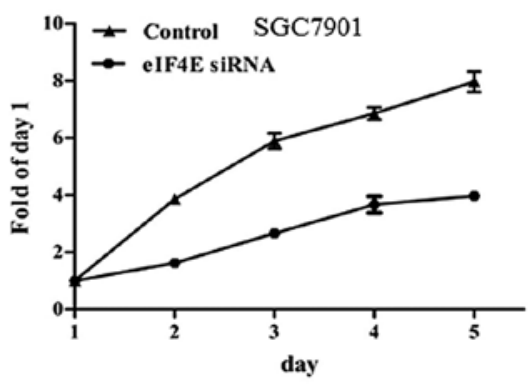

F

Blank Control siRNA
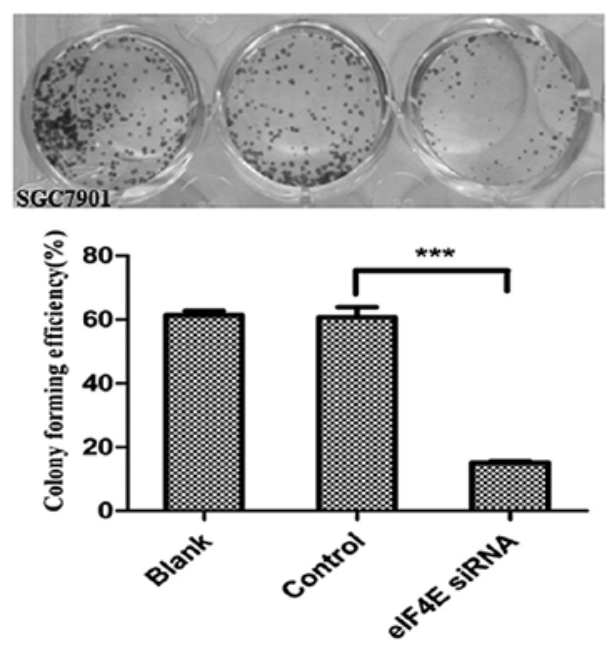

Figure 3. Silencing eIF4E reduces the growth of SGC7901 and MGC803 cells. (A and B) Western blotting and qRT-PCR showed that the level of eIF4E in SGC7901 and MGC803 cells with RNA inference was substantially decreased in comparison with those transfected with control siRNA (control). (C and D) SRB assay was used to evaluate the cell viability from days 1-5. (E and F) The results of colony formation assay for the cells transfected with eIF4E siRNA (siRNA) and control siRNA (control) groups and untreated cell lines (blank) and the quantification of colony formation assay. (E) MGC803 and (F) SGC7901 cells were treated with $100 \mathrm{nmol} / \mathrm{l}$ eIF4E siRNA and control siRNA as indicated for 12 days and subjected to colony formation assay. All data in the different experimental groups are expressed as the means $\pm \mathrm{SD},{ }^{* *} \mathrm{P}<0.01 ;{ }^{* * * *} \mathrm{P}<0.001$.

of gastric cancer cells through regulating the Akt signaling pathway. After $24 \mathrm{~h}$ of treatment, perifosine significantly downregulated the level of eIF4E mRNA in SGC7901 cells $(\mathrm{P}<0.05)$, but showed a mild change and not in a time- and dose-dependent manner (Fig. 4A and B); perifosine $(0.25$, $0.5,0.75$ and $1.0 \mu \mathrm{mol} / \mathrm{l}$ ) produced the dose-dependently decreased expression of T-eIF4E and p-eIF4E in SGC7901 cells (Fig. 4D and E). In MGC803 cells, the expression of eIF4E mRNA was significantly downregulated by perifosine in a dose-dependent manner (Fig. 4C). Furthermore, we also examined the expression of total Akt and p-Akt (s473), and the T-eIF4E and p-eIF4E (s209) were also decreased in a doseand time-dependent manner in MGC803 cells (Fig. 4F and G).
These results indicated that eIF4E could be a target of perifosine in gastric cancer, and the Akt/eIF4E signaling pathway was involved in the regulation mechanism of perifosine.

Perifosine inhibits the growth of gastric cancer cells in a dosedependent manner. Perifosine dose-dependently decreased the viability of gastric cancer cells at the concentrations ranging from 0.625 to $15 \mu \mathrm{mol} / \mathrm{l}$ in a 3-day SRB assay (Fig. 5A). Among these cell lines, SGC7901 was the most sensitive to perifosine treatment with an $\mathrm{IC}_{50}$ of $0.36 \mu \mathrm{mol} / 1$. The $\mathrm{IC}_{50}$ of MGC803, MKN45 and AGS cells was 5.18, 11.65 and $4.0 \mu \mathrm{mol} / 1 \mathrm{respec}-$ tively, which indicated that perifosine inhibited the growth of gastric cancer cells with different sensitivity. In addition, peri- 
A

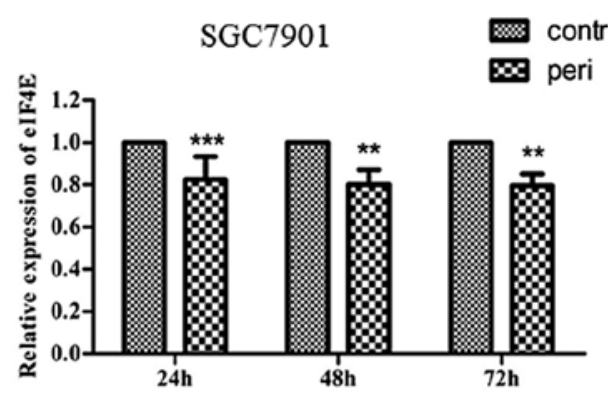

$\mathrm{C}$
B

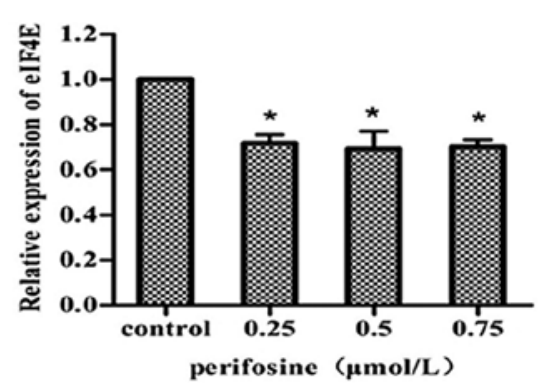

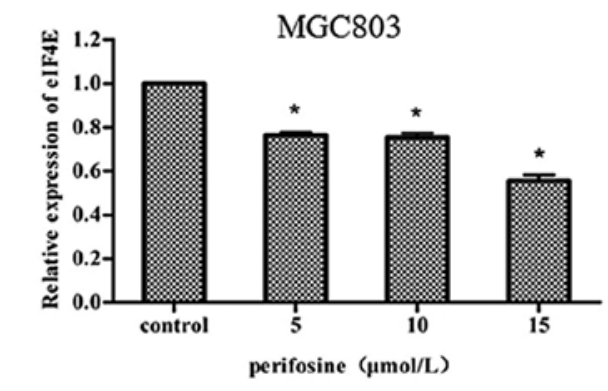

D

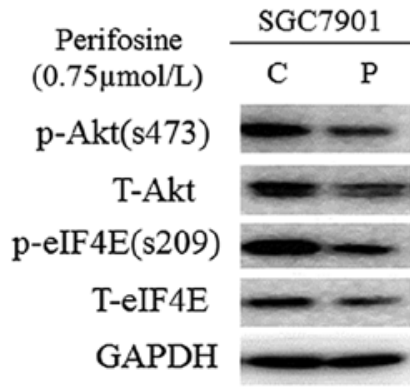

F

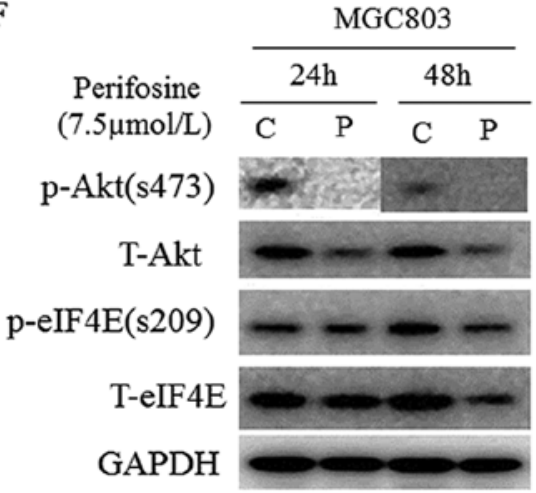

$\mathrm{E}$

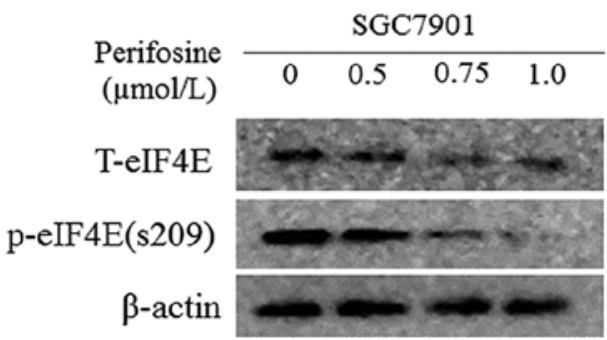

G

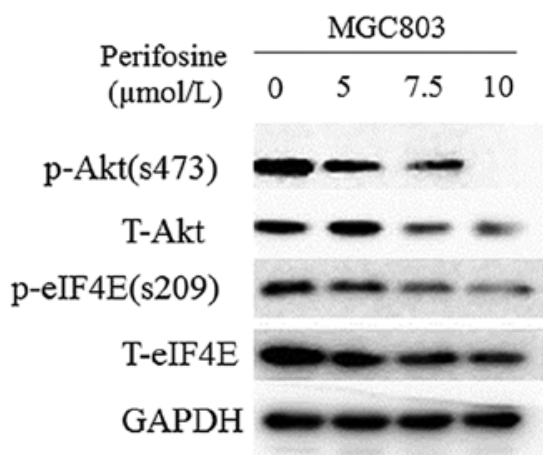

Figure 4. Perifosine decreases the mRNA levels of eIF4E and inhibits the Akt/eIF4E pathway while reducing total eIF4E and p-eIF4E amounts. (A and B) The mRNA levels of eIF4E in SGC7901 cells treated with $0.75 \mu \mathrm{mol} / 1$ perifosine for $24 \mathrm{~h}$ or exposed to the indicated concentration of perifosine for $24 \mathrm{~h}$ in qRT-PCR assay. (C) The mRNA levels of eIF4E in MGC803 cells exposed to the indicated concentration of perifosine for $24 \mathrm{~h}$ in qRT-PCR assay. (D and E) SGC7901 cells were treated with $0.75 \mu \mathrm{mol} / 1$ perifosine for $24 \mathrm{~h}$ or exposed to different concentrations of perifosine ranging from 0.5-1.5 $\mu \mathrm{mol} / 1$ for $24 \mathrm{~h}$. (F and G) MGC803 cells were treated with $7.5 \mu \mathrm{mol} / 1$ perifosine for 24 and $48 \mathrm{~h}$ or incubated with different concentrations of perifosine ranging from 5-15 $\mu \mathrm{mol} / \mathrm{l}$ for $24 \mathrm{~h}$. Total protein was extracted, and the expression of p-Akt (s473), total Akt (T-Akt), total eIF4E (T-eIF4E), p-eIF4E (s209) were analyzed by western blot analysis. All results are representative of three independent experiments and are expressed as the means $\pm \mathrm{SD},{ }^{*} \mathrm{P}<0.05 ;{ }^{* *} \mathrm{P}<0.01 ;{ }^{* * *} \mathrm{P}<0.001$.

fosine dose-dependently substantially decreased the ability of MGC803 (Fig. 5B) and SGC7901 (Fig. 5C) cells to form colonies in a dose-dependent manner.

Co-targeting eIF4E enhances the inhibitory effect on gastric cancer SGC7901 and MGC803 cells. Since perifosine inhibited the growth of gastric cancer cells, we used RNA interference to block eIF4E expression and then evaluated the combined effect of eIF4E siRNA and perifosine on gastric cancer cell lines. The results of qRT-PCR assay showed eIF4E siRNA had a very high inhibitory effect on eIF4e in SGC7901 and MGC803 cells (Fig. 3A). Western blotting also confirmed the knockdown rate of eIF4E (Fig. 3B). The combined treatment of eIF4E siRNA and perifosine enhanced the inhibitory effect on gastric cancer cell growth (Fig. 6A and B). We further examined the eIF4E signaling pathway regulated by perifo- 

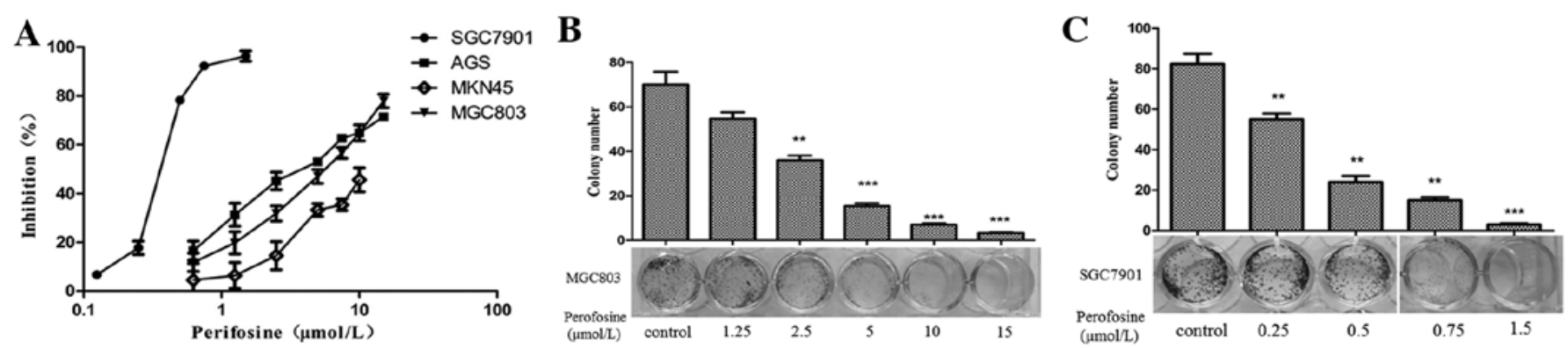

Figure 5. Perifosine inhibits the proliferation of gastric cancer cells. (A) SGC7901, MGC803, AGS and MKN45 cells were treated with perifosine at different concentrations for $72 \mathrm{~h}$. The cell viability was determined by SRB assay. (B and C) Perifosine inhibited the colony formation of (B) MGC 803 and (C) SGC7901 cells. The cells were treated with perifosine dose-dependently for 12 days and subjected to colony formation assay. All data in the different experimental groups are expressed as the means $\pm \mathrm{SD},{ }^{* *} \mathrm{P}<0.01 ;{ }^{* * * *} \mathrm{P}<0.001$.

A

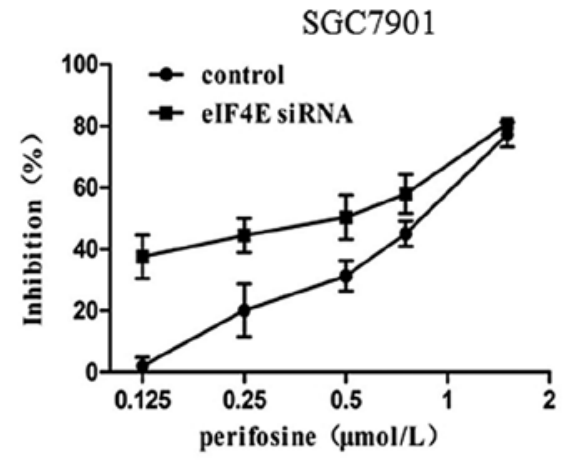

$\mathrm{C}$

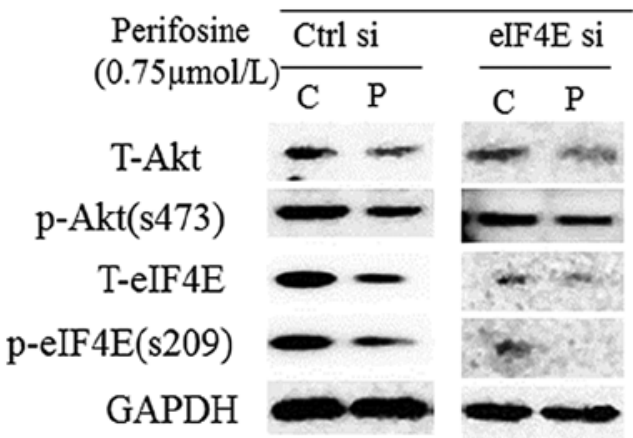

B

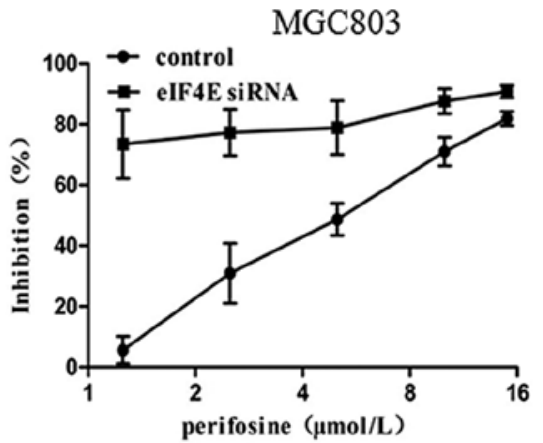

D

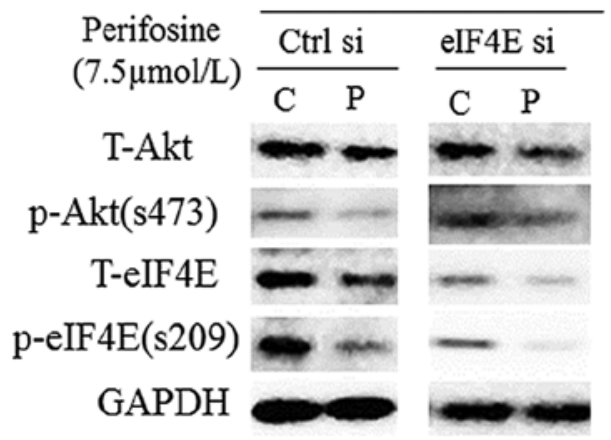

Figure 6. The combination of eIF4E gene-silencing and perifosine treatment causes significant inhibitory effect on the growth of gastric cancer cells. (A and B) Twenty-four hours after transfection with eIF4E siRNA, (A) SGC7901 and (B) MGC803 cells were treated with perifosine for 72 h, and the viability loss after the combined treatment of eIF4E siRNA and perifosine were measured by SRB assay. (C and D) SGC7901 and MGC803 cells were transfected with eIF4E siRNA (eIF4E si) or control siRNA (Ctrl si), respectively. Twenty-four hours after transfection, the cells were treated with or without perifosine for 48 h. Total protein lysates were collected and subjected to western blot analysis. All results are representative of three independent experiments and are expressed as the means $\pm \mathrm{SD}$. C, control; P, perifosine.

sine. After SGC7901 and MGC803 cells transfected with eIF4E siRNA or a control siRNA were treated with perifosine, eIF4E expression was further downregulated (Fig. 6C and D). The results suggested the combination of eIF4E siRNA and perifosine may represent an effective therapy against gastric cancer.

\section{Discussion}

Abnormal eIF4E activity is present in a large spectrum of human malignancies. eIF4E overexpression has been commonly seen in the prostate, breast, stomach, colon, lung, skin and hematopoietic system. p-eIF4E has oncogenic potential in human cancer $(20,21)$. However, little is known about the relationship between the eIF4E gene and gastric cancer progression. In the current study, we found that the expression levels of T-eIF4E and p-eIF4E were upregulated in gastric cancer samples. Further analysis showed that T-eIF4E expression was related to cancer vascular invasion, suggesting $\mathrm{T}$-eIF4E promoted the progression of gastric cancer. However, we did not find a relationship between p-eIF4E expression and any clinical characteristics of the patients. Meanwhile, due to limited samples, the relationship between T-eIF4E and p-eIF4E expression and the malignancy of human tumors remains to be further determined. These data indicate that the eIF4E gene may be an attractive target for gastric cancer therapy. 
Translation activation is critical for cancer cell growth and survival. Therefore, translation is a rational target for novel cancer therapeutics. Translation initiation is largely dependent on eIF4E activity. According to research on different types of tumors, eIF4E can be regulated by the PI3K/mTOR, MAPK/MNK signaling pathways at multiple levels, such as transcription, serine 209 phosphorylation, as well as the inhibitory interaction between binding proteins (4EBP) $(22,23)$. Furthermore, the difference between eIF4E activation and response to eIF4E siRNA suggests varying influence of the Akt pathway depending on cell types. In this study, we found that eIF4E was overexpressed in gastric cell lines and knockdown of eIF4E in MGC803 and SGC7901 cells through RNA interference significantly inhibited in vitro proliferation. Therefore, eIF4E may play an important role in gastric cancer, and clarification of the eIF4E signaling pathway is urgently required.

Perifosine, an alkylphospholipid with antitumor activity in both preclinical and clinical studies, inhibits Akt through targeting its pleckstrin homology $(\mathrm{PH})$ domain and interferes with its recruitment to the plasma member and subsequent phosphorylation and activation (24). In the present study, we investigated the effect of perifosine on human gastric cancer SGC7901, MGC803, AGS and MKN45 cells. Our results indicate that perifosine treatment has a significant cytotoxic effect on SGC7901, MGC803, AGS and MKN45 cells in a dosedependent manner, which is associated with rapidly decreased Akt activation as assessed by the quantification of Ser473 phosphorylation and the decreased T-eIF4E and p-eIF4E expression. Previous studies have indicated that eIF4E is one of the proposed downstream mediators in the Akt signaling pathway and regulated by Akt. These findings indicate that perifosine may downregulate the expression of eIF4E through Akt inhibition (25).

We first observed that perifosine downregulated T-eIF4E and p-eIF4E expression and inhibited the Akt/eIF4E signaling pathway in gastric cancer. We also noted less changed eIF4E amounts at the mRNA level in SGC7901 cells than at the protein level, which suggested the involvement of other mechanisms in addition to transcriptional regulation. Furthermore, enhanced eIF4E level has been demonstrated to increase the expression of proteins that contribute to tumor development, including growth factors such as c-Myc and cyclin D1, and angiogenesis factors such as VEGF and FGF-2. Perifosine inhibited the proliferation of gastric cancer cells through decreasing eIF4E levels. Further studies on whether the inhibition of eIF4E by perifosine also inhibits the expression of other downstream targets that are necessary for oncogenesis and cancer progression are warranted.

After identifying that the eIF4E signaling pathway was regulated by perifosine, we also found that the combination of eIF4E gene silencing and perifosine treatment could significantly increase the growth inhibition efficacy in human gastric cancer cells in comparison with eIF4E gene silencing or perifosine treatment alone. Cancer cells tend to be more highly dependent on translation than normal tissues. Targeting eIF4E appears to be an attractive anticancer strategy for chronic myeloid leukemia and endometrial adenocarcinoma $(5,7,18)$. Increasing evidence supports that inhibited eIF4E can cause more inhibitory effects on drug-induced growth (8). Therefore, suppressing translation by targeting eIF4E with RNA interfer- ence may play a more important role in perifosine-induced growth repression. The result may suggest a possible strategy of combined eIF4E knockdown.

In conclusion, the current results showed that the expression of T-eIF4E and p-eIF4E was increased in gastric cancer tissues and cell lines. Downregulation of eIF4E significantly suppressed the proliferation of gastric cancer cells. Moreover, perifosine exerted its inhibitory effect on the regulation of growth and survival through the eIF4E signaling pathway and the combination of eIF4E gene silencing and perifosine was more effective than eIF4E gene silencing or perifosine alone. Further research might focus on a specific mechanism through which eIF4E regulates the growth of gastric cancer and may confirm the potential effectiveness of eIF4E as a therapeutic target of gastric cancer in clinical practice.

\section{Acknowledgements}

This study was supported by the Jiangsu Province's Key Provincial Talents Program, China (RC2011059) (L.Y.), and the 'Six Talents Peak' Project of Jiangsu Province (2012) (L.Y.), the Priority Academic Program Development of Jiangsu Higher Education Institutions (PAPD), and the National Natural Science Foundation of China (nos. 30873099, 81102458 and 81172004) (X.W.). The authors also thank Professor Xuerong Wang of Nanjing Medical University for the valuable comments on this study.

\section{References}

1. Avdulov S, Li S, Michalek V, et al: Activation of translation complex eIF4F is essential for the genesis and maintenance of the malignant phenotype in human mammary epithelial cells. Cancer Cell 5: 553-563, 2004.

2. Chen CN, Hsieh FJ, Cheng YM, Lee PH and Chang KJ: Expression of eukaryotic initiation factor $4 \mathrm{E}$ in gastric adenocarcinoma and its association with clinical outcome. J Surg Oncol 86: 22-27, 2004.

3. De Benedetti A and Graff JR: eIF-4E expression and its role in malignancies and metastases. Oncogene 23: 3189-3199, 2004.

4. Dong K, Wang R, Wang X, et al: Tumor-specific RNAi targeting eIF4E suppresses tumor growth, induces apoptosis and enhances cisplatin cytotoxicity in human breast carcinoma cells. Breast Cancer Res Treat 113: 443-456, 2009.

5. Choi CH, Lee JS, Kim SR, et al: Direct inhibition of eIF4E reduced cell growth in endometrial adenocarcinoma. J Cancer Res Clin Oncol 137: 463-469, 2011.

6. Herbert TP, Fahraeus R, Prescott A, Lane DP and Proud CG: Rapid induction of apoptosis mediated by peptides that bind initiation factor eIF4E. Curr Biol 10: 793-796, 2000.

7. Ge Y, Cheng R, Zhou Y, et al: Cryptotanshinone induces cell cycle arrest and apoptosis of multidrug resistant human chronic myeloid leukemia cells by inhibiting the activity of eukaryotic initiation factor 4E. Mol Cell Biochem 368: 17-25, 2012.

8. Zhou FF, Yan M, Guo GF, et al: Knockdown of eIF4E suppresses cell growth and migration, enhances chemosensitivity and correlates with increase in $\mathrm{Bax} / \mathrm{Bcl}-2$ ratio in triple-negative breast cancer cells. Med Oncol 28: 1302-1307, 2011.

9. Gills JJ and Dennis PA: Perifosine: update on a novel Akt inhibitor. Curr Oncol Rep 11: 102-110, 2009.

10. Elrod HA, Lin YD, Yue P, et al: The alkylphospholipid perifosine induces apoptosis of human lung cancer cells requiring inhibition of Akt and activation of the extrinsic apoptotic pathway. Mol Cancer Ther 6: 2029-2038, 2007.

11. Papa V, Tazzari PL, Chiarini F, et al: Proapoptotic activity and chemosensitizing effect of the novel Akt inhibitor perifosine in acute myelogenous leukemia cells. Leukemia 22: 147-160, 2008.

12. Tazzari PL, Tabellini G, Ricci F, et al: Synergistic proapoptotic activity of recombinant TRAIL plus the Akt inhibitor perifosine in acute myelogenous leukemia cells. Cancer Res 68: 9394-9403, 2008. 
13. Leighl NB, Dent S, Clemons M, et al: A Phase 2 study of perifosine in advanced or metastatic breast cancer. Breast Cancer Res Treat 108: 87-92, 2008.

14. Ghobrial IM, Roccaro A, Hong F, et al: Clinical and translational studies of a phase II trial of the novel oral Akt inhibitor perifosine in relapsed or relapsed/refractory Waldenstrom's macroglobulinemia. Clin Cancer Res 16: 1033-1041, 2010.

15. Cho DC, Hutson TE, Samlowski W, et al: Two phase 2 trials of the novel Akt inhibitor perifosine in patients with advanced renal cell carcinoma after progression on vascular endothelial growth factor-targeted therapy. Cancer 118: 6055-6062, 2012.

16. Ma Z, Zhu L, Luo X, Zhai S, Li P and Wang X: Perifosine enhances mTORC1-targeted cancer therapy by activation of GSK3 $\beta$ in NSCLC cells. Cancer Biol Ther 13: 1009-1017, 2012.

17. Wang S, Rosenwald IB, Hutzler MJ, et al: Expression of the eukaryotic translation initiation factors 4E and 2alpha in nonHodgkin's lymphomas. Am J Pathol 155: 247-255, 1999.

18. Muta D, Makino K, Nakamura H, Yano S, Kudo M and Kuratsu J: Inhibition of eIF4E phosphorylation reduces cell growth and proliferation in primary central nervous system lymphoma cells. J Neurooncol 101: 33-39, 2011.

19. Soni A, Akcakanat A, Singh G, et al: eIF4E knockdown decreases breast cancer cell growth without activating Akt signaling. Mol Cancer Ther 7: 1782-1788, 2008
20. Fan SQ, Ramalingam SS, Kauh J, Xu ZH, Khuri FR and Sun SY: Phosphorylated eukaryotic translation initiation factor 4 (eIF4E) is elevated in human cancer tissues. Cancer Biol Ther 8: 1463-1469, 2009.

21. Wheater MJ, Johnson PW and Blaydes JP: The role of MNK proteins and eIF4E phosphorylation in breast cancer cell proliferation and survival. Cancer Biol Ther 10: 728-735, 2010.

22. Fischer PM: Cap in hand: targeting eIF4E. Cell Cycle 8: 2535-2541, 2009.

23. Jia Y, Polunovsky V, Bitterman PB and Wagner CR: Cap-dependent translation initiation factor eIF4E: an emerging anticancer drug target. Med Res Rev 32: 786-814, 2012.

24. Hideshima T, Catley L, Yasui H, et al: Perifosine, an oral bioactive novel alkylphospholipid, inhibits Akt and induces in vitro and in vivo cytotoxicity in human multiple myeloma cells. Blood 107: 4053-4062, 2006.

25. Yoshizawa A, Fukuoka J, Shimizu S, et al: Overexpression of phospho-eIF4E is associated with survival through AKT pathway in non-small cell lung cancer. Clin Cancer Res 16: 240-248, 2010. 